\title{
Implementation and Analysis of Multiple Polar Harmonic Equations in a Rotatory Two Dimensional Graphical Plane for Thumb Pore Identification
}

\author{
Anjali Rana \\ BBSBEC, Fatehgarh Sahib \\ Punjab Technical University, Jalandhar, India
}

\author{
Ishpreet Virk \\ Assistant Professor CSE Dept. \\ BBSBEC, Fatehgarh Sahib \\ Punjab Technical University, Jalandhar, India
}

\begin{abstract}
Polar harmonic transforms (PHTs) are a set of 2D transforms, which are based on a set of orthogonal projection bases, to generate a set of features which are invariant to rotation. PHTs represent a set of transforms whose kernels are basic waves and harmonic in nature. In this paper, Polar harmonic transforms (PHTs) are analyzed for rotation invariance and two equations are compared, namely, Polar Complex Exponential Transform (PCET) and Polar Cosine Transform (PCT), based on different parameters like Euclidean distance, False Rejection Rate (FRR) and False Acceptance Rate (FAR). Out of these two equations, Polar Cosine Transform (PCT) shows better results. The polar harmonic equations perform well in presence of rotation. Orthogonal kernels of PHTs are more effective in terms of information compactness and minimal information redundancy.
\end{abstract}

\section{Keywords}

Polar harmonic transforms, Polar complex exponential transform, Polar cosine transform, Fingerprint recognition and matching, Poroscopy, marker controlled watershed Segmentation, False acceptance rate, False rejection rate, Euclidean distance.

\section{INTRODUCTION}

One of the most significant problem in image retrieval and pattern recognition is rotation invariant pattern representation. This arises from the fact that in many real world applications images should be considered to be the same even if they are rotated. Many types of moment and transform have been proposed for feature extraction and invariant image representation. Among them are Zernike Moments (ZMs), which is often mentioned together with Pseudo-Zernike Moments (PZMs) and orthogonal Fourier-Mellin moments These moments are popular and are used in many real world applications like character recognition and multi-spectral texture analysis[15]. But computations of their kernels involve a number of factorial terms and suffer from numerical instability. However, Polar Harmonic Transforms (PHTs) have no numerical instability.

In this paper, a set of transforms, called Polar Harmonic Transforms (PHTs) are introduced. These transforms can be used to generate rotation invariant features. PHTs consists of three different transforms, namely, Polar Complex Exponential Transform (PCET), Polar Cosine Transform (PCT), and Polar Sine Transform (PST). The PHTs are characterized by low-time complexity and numerical stability. The kernel functions of PHTs are orthogonal. Orthogonality of kernel means that an image is projected onto a set of pairwise orthogonal axes, and hence the classifier can be simple[1]. The features based on orthogonal kernels are more effective in terms of information compactness and minimal information redundancy. Owing to these characteristics, PHTs are well suited for various applications such as Fingerprint classification[3], Pattern recognition[11], Character recognition[15], Image reconstruction[1], etc.

The organization of this paper is as follows. The basic theories of Polar Harmonic Transforms (PHTs) with Polar Exponential Cosine Transforms (PCET) and Polar Cosine Transforms (PCT) including mathematics descriptions are provided in section II. The brief explanation about fingerprint, Pore Extraction, Fingerprint recognition and fingerprint matching is given in Section III. In Section IV, the parameters Euclidean distance, False Rejection Rate (FRR) and False Acceptance Rate (FAR), are explained. They are used for the comparison of the two transforms, PCET and PCT. Section V explains the methodology used for the implementation. In section VI, the performance of PCET and PCT is compared based on parameters. Section VII concludes this study.

\section{POLAR HARMONIC TRANSFORMS}

Polar harmonic transforms (PHTs) is set of three different transforms Polar Complex Exponential Transform (PCET), Polar Cosine Transform (PCT) and Polar Sine Transform (PST), whose kernels are basic waves and harmonic in nature. PHTs have identical mathematical representation, the difference lies in the radial part of the kernel function. PHTs are defined on a unit circle.

For an image $f(r, \theta)$, the PCET of order $n$ with repetition $l,|n|,|l|=0,1,2, \ldots, \infty$, is defined as :

$M_{n l}=\frac{1}{\pi} \int_{0}^{2 \pi} \int_{0}^{1}\left[H_{n l}(r, \theta)\right]^{*} f(r, \theta) r d r d \theta$

where the basis function $H_{n l}(r, \theta)$ consists of a radial component $R_{n}(r)$ and a circular component $e^{i l \theta}$ :

$$
H_{n l}(r, \theta)=R_{n}(r) e^{i l \theta}
$$

with $R_{n}(r)$ in the following form

$R_{n}(r)=e^{i 2 \pi n r^{2}}$

similarly, PCT and PST is defined as:

$$
M_{n l}^{c}=\Omega_{n} \int_{0}^{2 \pi} \int_{0}^{1}\left[H_{n l}^{c}(r, \theta)\right]^{*} f(r, \theta) r d r d \theta
$$




$$
\begin{gathered}
M_{n l}^{s}=\Omega_{n} \int_{0}^{2 \pi} \int_{0}^{1}\left[H_{n l}^{s}(r, \theta)\right]^{*} f(r, \theta) r d r d \theta \\
n,|l|=0,1,2, \ldots, \infty,
\end{gathered}
$$

with their kernels

$$
\begin{aligned}
& H_{n l}^{c}=R_{n}^{c}(r) e^{i l \theta}=\cos \left(\pi n r^{2}\right) e^{i l \theta} \\
& H_{n l}^{s}=R_{n}^{S}(r) e^{i l \theta}=\sin \left(\pi n r^{2}\right) e^{i l \theta}
\end{aligned}
$$

and

$$
\Omega_{n}=\left\{\begin{array}{l}
\frac{1}{\pi}, n=0 \\
\frac{2}{\pi}, n \neq 0
\end{array}\right.
$$

PCET and PCT are defined on a unit circle. $M_{n l}$ and $M_{n l}^{c}$ are rotation invariant.

\section{FINGERPRINT}

Fingerprints are the imprints formed by the friction ridges and furrows of the skin and thumbs. These ridges and furrows present good similarities in each small local window, like parallelism and average width. The fingerprints have long been used for its immutability and individuality. Immutability means permanent and unchanging character of the pattern on each finger[6] and individuality means the uniqueness of ridge details across individuals. Fingerprints are considered to be one of the most legitimate proof of evidence in courts of law and have widely been used for personnel recognition in the commercial and forensic areas because of its uniqueness and immutability.

\subsection{Pore Extraction}

Marker Controlled Watershed Segmentation method is used for pore extraction from thumb image[17]. The algorithm creates foreground and background markers using Morphological image reconstructions. The Watershed transform of the marker-modified gradient fingerprint image is computed. Pores are extracted from the image, by superimposing the watershed ridge lines on the original thumb image[18]

\subsection{Fingerprint Recognition}

Fingerprint recognition is a way of matching or verifying a fingerprint against another fingerprint to determine if the impressions are same. Fingerprint recognition based on local robust features is considered to be the best because one is able to perform person recognition in presence of rotated fingerprint images [6],[12].

\subsection{Fingerprint Matching}

Fingerprint matching is a process of comparing an original image with the test image. One of the well known technique used for fingerprint recognition is minutiae-based. Fingerprint matching based on minutiae features is been used by many researchers, this technique often assumes that the two fingerprints to be matched are of approximately same size[16]. However, this assumption is not valid in general. The two images with different orientation may fail to match in minutiae-based due to relative change in their minutiae locations. To overcome this problem, a set of Polar Harmonic Transforms (PHTs) are introduced in this paper because they are invariant to rotation. These transforms are used for fingerprint matching, based on sweat pores[18].

\section{PARAMETERS USED}

In this paper the following three parameters are used for comparison. First one is Euclidean Distance (ED); second one is False Rejection Rate (FRR); and third is False Acceptance Rate (FAR).

\subsection{Euclidean Distance (ED)}

In a Euclidean plane the distance between two points $\boldsymbol{p}=\left(p_{1}, p_{2}\right)$ and $\boldsymbol{q}=\left(q_{1}, q_{2}\right)$ is given by :

$d(p, q)=\sqrt{\left(p_{1}-q_{1}\right)^{2}+\left(p_{2}-q_{2}\right)^{2}}$

Alternatively, if the polar coordinates of the point $\boldsymbol{p}$ are $\left(r_{1}, \theta_{1}\right)$ and those of $\boldsymbol{q}$ are $\left(r_{2}, \theta_{2}\right)$ then the distance between the points is :

$d(p, q)=\sqrt{r_{1}^{2}+r_{2}^{2}-2 r_{1} r_{2} \cos \left(\theta_{1}-\theta_{2}\right)}$

Here, polar coordinates have been used to find the Euclidean distance (ED).

\subsection{False Rejection Rate (FRR)}

From an image database, each test image is rotated by various angles and is matched against the original image to compute the False Rejection Rate[6]. So, False Rejection Rate (FRR) is the ratio of the number of false rejections divided by the number of identification attempts. FRR is also known as False Non Match Rate or Type I error.

\subsection{False Acceptance Rate (FAR)}

Again from an image database, each test image is rotated by various angles and is matched against the original image to compute the False Acceptance Rate[6]. So, False Acceptance Rate (FAR) is the ratio of the number of false acceptances divided by the number of identification attempts. FRR is also known as Non Match Rate or Type II error.

\section{METHODOLOGY}

This section describes the methodology used for the implementation of proposed work.

Steps involved:

1. Load an image of thumb from the database.

2. Resize the image to reduce pixels to $150 \times 150$ pixels.

3. Segment image using watershed transform directly on the gradient magnitude.

4. Extract pores from thumb image with Marker controlled Watershed Algorithm.

5. Crop image to get the region of interest.

6. Apply Polar Complex Exponential Transform (PCET) on the thumb image.

7. Apply histogram and save the image (image A).

8. Take the cropped image of thumb from step 2 .

9. Again apply PCET equation on cropped image.

10. Rotate the image at the specified angles.

11. Apply histogram and save the new image (image B).

12. Calculate the Euclidean distance between the two images, i.e., image A and image B.

13. Loop through steps 9-12 to see the Euclidean distance at various rotated angles.

14. Fingerprint Matching-If Euclidean distance is less than or equal to the set threshold value, then the result is matched else not matched.

15. Repeat steps $1-14$ using PCT.

The lower the Euclidean distance, the more accurately matched the image is. 


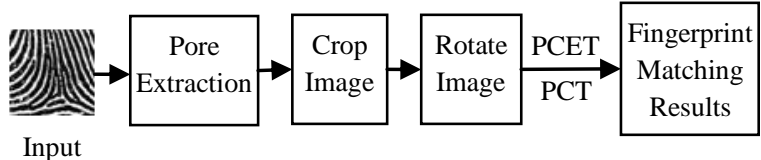

Image

Fig 1. Block Diagram of fingerprint matching system.

Figure 1 shows the steps explained above. This approach will be used to compare the effectiveness of the two transforms.

\section{RESULTS AND DISCUSSION}

The performance of the proposed Polar Complex Exponential Transform (PCET) and Polar Cosine Transform (PCT) is validated through comparative experiments using various images. One hundred thumb images gathered from fifty subjects are used in the experiments. Few sample images from the database are shown in Figure 2.
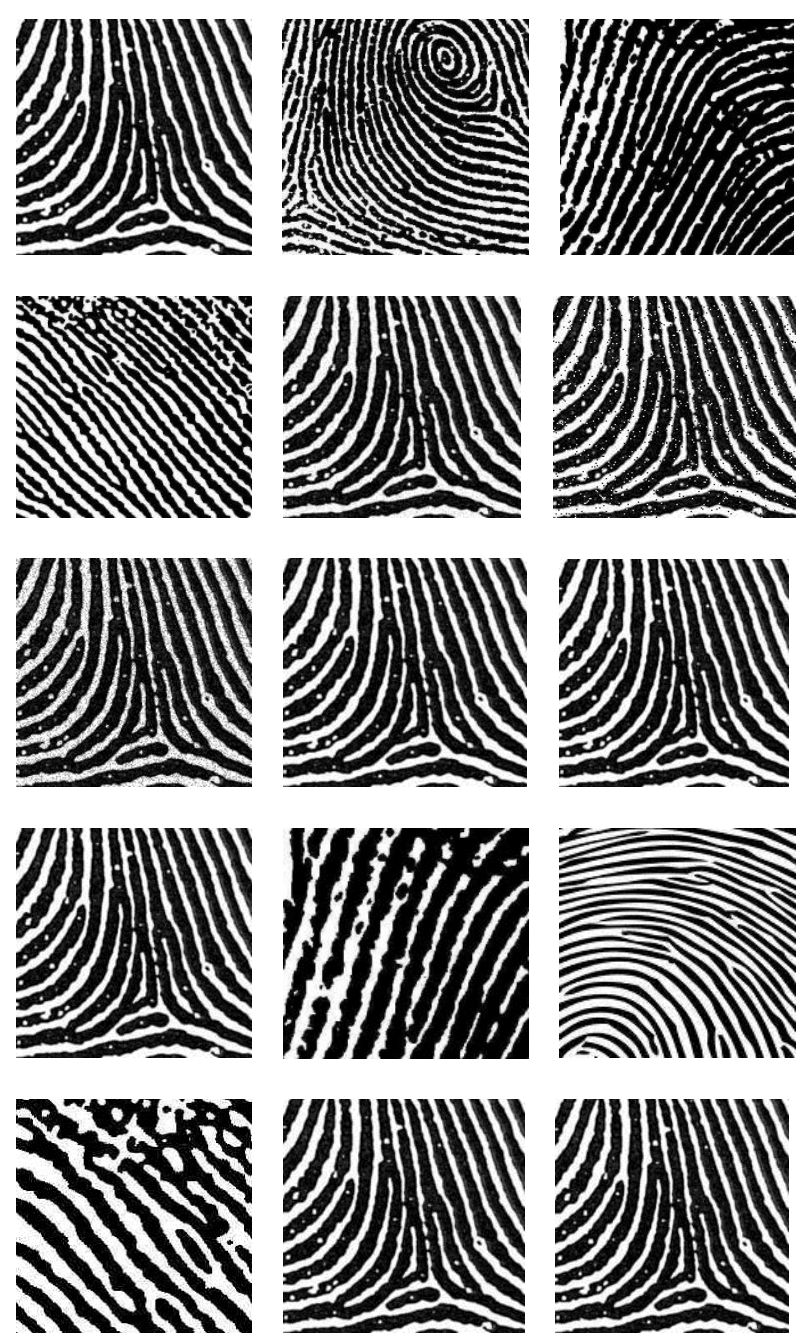

Fig 2: Thumb image samples from database.

The methodology mentioned in Section $\mathrm{V}$ is implemented, for both transforms, by taking one base image and five test images. Three images are matching and two are nonmatching. All the test images are rotated by ten degrees is matched against the base image. Then the Euclidean distance between the base and rotated image is calculated. This is carried out for fifteen angles. FRR and FAR is also calculated for each entry. Table 1 and Table 2 show the results.
Table 1. Shows the parameter results of PCET applied on test images.

\begin{tabular}{|c|c|c|c|c|c|c|c|c|}
\hline \multirow{3}{*}{$\begin{array}{l}\mathbf{P} \\
\mathbf{H} \\
\mathbf{T}\end{array}$} & \multirow{3}{*}{$\begin{array}{c}\text { Angles } \\
\text { of } \\
\text { Rotation }\end{array}$} & \multicolumn{7}{|c|}{ Parameters } \\
\hline & & \multicolumn{5}{|c|}{ Euclidean Distance (ED) } & \multirow{2}{*}{$\begin{array}{l}\mathbf{F} \\
\mathbf{R} \\
\mathbf{R} \\
\end{array}$} & \multirow{2}{*}{$\begin{array}{l}\mathbf{F} \\
\mathbf{A} \\
\mathbf{R}\end{array}$} \\
\hline & & $\mathbf{E D}_{1}$ & $\mathbf{E D}_{2}$ & $\mathbf{E D}_{3}$ & $\mathbf{E D}_{4}$ & $\mathbf{E D}_{5}$ & & \\
\hline \multirow{15}{*}{$\begin{array}{l}\mathbf{P} \\
\mathbf{C} \\
\mathbf{E} \\
\mathbf{T}\end{array}$} & 10 & 1.79 & 2.82 & 0.79 & 45.6 & 221.1 & \multirow{15}{*}{$\begin{array}{l}\mathrm{N} \\
\mathrm{I} \\
\mathrm{L}\end{array}$} & \multirow{15}{*}{$\begin{array}{l}\mathrm{N} \\
\mathrm{I} \\
\mathrm{L}\end{array}$} \\
\hline & 20 & 1.76 & 2.12 & 1.14 & 48.0 & 222.2 & & \\
\hline & 30 & 1.59 & 1.06 & 1.06 & 45.4 & 220.8 & & \\
\hline & 40 & 0.88 & 2.47 & 2.03 & 43.8 & 223.0 & & \\
\hline & 50 & 3.18 & 0.61 & 2.12 & 45.6 & 216.4 & & \\
\hline & 60 & 1.23 & 2.56 & 0.53 & 47.1 & 220.3 & & \\
\hline & 70 & 1.32 & 1.59 & 0.35 & 46.4 & 218.4 & & \\
\hline & 80 & 0.35 & 2.65 & 0.08 & 47.7 & 220.9 & & \\
\hline & 90 & 0.61 & 2.38 & 0.97 & 45.6 & 214.0 & & \\
\hline & 100 & 0.44 & 1.59 & 1.14 & 47.0 & 220.7 & & \\
\hline & 110 & 1.32 & 0.08 & 1.41 & 45.4 & 218.3 & & \\
\hline & 120 & 1.41 & 2.56 & 0.44 & 47.1 & 218.6 & & \\
\hline & 130 & 1.67 & 1.23 & 2.47 & 43.4 & 216.4 & & \\
\hline & 140 & 2.20 & 0.26 & 2.20 & 46.4 & 223.3 & & \\
\hline & 150 & 0.08 & 2.29 & 0.97 & 45.8 & 224.0 & & \\
\hline
\end{tabular}

Table 2. Shows the parameter results of PCT applied on test images.

\begin{tabular}{|c|c|c|c|c|c|c|c|c|}
\hline \multirow{3}{*}{$\begin{array}{l}\mathbf{P} \\
\mathbf{H} \\
\mathbf{T}\end{array}$} & \multirow{3}{*}{$\begin{array}{l}\text { Angles } \\
\text { of } \\
\text { Rotation }\end{array}$} & \multicolumn{7}{|c|}{ Parameters } \\
\hline & & \multicolumn{5}{|c|}{ Euclidean Distance (ED) } & \multirow{2}{*}{$\begin{array}{l}\mathbf{F} \\
\mathbf{R} \\
\mathbf{R}\end{array}$} & \multirow{2}{*}{$\begin{array}{l}\mathbf{F} \\
\mathbf{A} \\
\mathbf{R}\end{array}$} \\
\hline & & $\mathbf{E D}_{1}$ & $\mathbf{E D}_{2}$ & $\mathbf{E D}_{3}$ & $\mathbf{E D}_{4}$ & $\mathbf{E D}_{5}$ & & \\
\hline \multirow{15}{*}{$\begin{array}{l}\mathbf{P} \\
\mathbf{C} \\
\mathbf{T}\end{array}$} & 10 & 1.32 & 0.35 & 0.35 & 31.0 & 141.5 & \multirow{15}{*}{$\begin{array}{l}\mathrm{N} \\
\mathrm{I}\end{array}$} & \multirow{15}{*}{$\begin{array}{l}\mathrm{N} \\
\mathrm{I} \\
\mathrm{L}\end{array}$} \\
\hline & 20 & 2.20 & 1.50 & 0.44 & 28.4 & 135.0 & & \\
\hline & 30 & 0.53 & 1.32 & 0.70 & 30.3 & 138.2 & & \\
\hline & 40 & 0.17 & 0.44 & 1.94 & 32.0 & 139.2 & & \\
\hline & 50 & 1.41 & 1.76 & 0.35 & 32.1 & 134.6 & & \\
\hline & 60 & 0.61 & 2.38 & 1.41 & 33.5 & 142.5 & & \\
\hline & 70 & 2.12 & 0.79 & 0.79 & 29.5 & 142.4 & & \\
\hline & 80 & 0.88 & 1.23 & 0.53 & 33.4 & 143.2 & & \\
\hline & 90 & 1.85 & 0.88 & 0.44 & 32.5 & 140.4 & & \\
\hline & 100 & 2.03 & 0.79 & 1.67 & 31.0 & 139.5 & & \\
\hline & 110 & 0.53 & 1.23 & 0.35 & 32.1 & 132.3 & & \\
\hline & 120 & 0.35 & 0.70 & 0.44 & 32.4 & 140.8 & & \\
\hline & 130 & 0.79 & 0.61 & 0.97 & 30.4 & 143.8 & & \\
\hline & 140 & 3.71 & 1.59 & 3.53 & 33.4 & 140.2 & & \\
\hline & 150 & 0.88 & 0.61 & 0.53 & 31.2 & 144.2 & & \\
\hline
\end{tabular}


Comparing the Euclidean distance values in Table 1 (PCET equation) and Table 2 (PCT equation), PCT shows better results. It is clear from Table 1 (PCET equation) and Table 2 (PCT equation) that, there is no test image, which is falsely accepted or rejected, as the FRR and FAR ratios are 0 . This shows that the proposed transforms, Polar Complex Exponential Transform (PCET) and Polar Cosine Transform (PCT) would be efficiently able to differentiate between genuine and imposter images.

The following graphs show the performance of the two transforms.

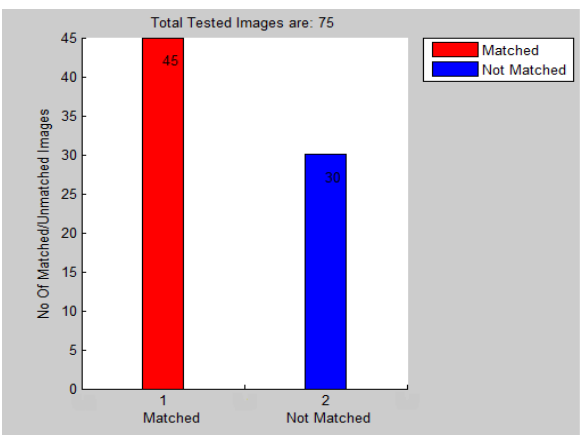

Fig 3:Matched and Unmatched Image score distribution

Figure 3 shows the score distribution of matched and unmatched images. As expected, both transforms showed invariance to image rotation.

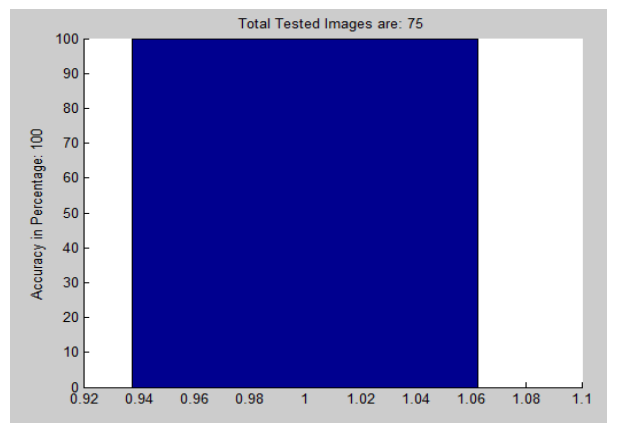

Fig 4:Graph shows the accuracy percentage of matched images

Figure 4 shows the accuracy percentage of matched images. It is clear from the graph that accuracy percentage is 100 , which means the proposed system matches all the test images efficiently, even after being rotated by various angles with the base image. Hence, the proposed transforms, PCET and PCT are rotation invariant.

\section{CONCLUSION}

In this paper, two Polar Harmonic Transform (PHT) equations have been proposed, Polar Complex Exponential Transform (PCET) and Polar Cosine Transform (PCT), in a rotatory two dimensional graphical plane for thumb pore identification and for rotation-invariant image representation. A comparison between PCET and PCT has been made. The Polar Cosine Transform (PCT) gives better results in comparison to Polar Complex Exponential Transform (PCET) because its Euclidean distance values are lower. PHTs find its applications in the area of image retrieval, image data mining, biomedical imaging, face detection and texture classification. Polar Harmonic Transforms (PHTs) are very fast and efficient. In future, a whole system can be made for testing live images.

\section{REFERENCES}

[1] Yap, P.T., Jiang, X., Kot, A.C. , July 2010. "Two Dimensional Polar Harmonic Transforms for Invariant Image Representation", IEEE Trans, Pattern Analysis and machine Intelligence, Vol. 32, N0. 7.

[2] Singh, C., Kaur, A., 2012, "Fast Computation of Polar Harmonic transforms", Springer.

[3] Liu, M., Jiang, X., Kot, A.C., Yap, P.T., 2011, "Application of Polar Harmonic Transforms to fingerprint classification", Handbook of Emerging Topics in Computer Vision and Applications , World Scientific Publishing, Singapore.

[4] Chander, K., Nath, R.: "Reducing Process Time for Fingerprint Identification System", International Journals of Biometric and Bioinformatics, vol. 3., Issue(1).

[5] Yang, Z., Kamata, S., 2011, "fast Polar Cosine Transform for Image Description", IAPR conference on Machine vision applications, June 13-15, Nara, Japan.

[6] Madhuri, Mishra, R., June 1012, "Fingerprint Recognition using Robust Local Features", IJARCSE, Vol. 2, Issue 6.

[7] Li, L., Li, S., Wang, G., Abnraham, A. , 2011, "An Evaluation on Circulatory Orthogonal Moments for Image Representation", International Conference on Information Science and Technology, March 26-28, Nanjing, Jiangsu, China.

[8] Yang, Z., Kamata, S.I. , Dec-2010, "fast Polar Harmonic Transforms", Int. Conf. Control, Automation Robotics and Vision, Singapore.

[9] Thai, V.H., Salvotore, T., 2012 "Fast Computation of orthogonal polar Harmonic Transforms", Int, Conf. on Pattern Recognition, November 11-15, Tsukuba, Japan.

[10] Singh, C., Upneja, R. , 2012, "Accuracy and Numerical Stability of High-Order Polar Harmonic Transforms", IET Image processing.

[11] Thai ,V.H., Salvatore, T., , 2011, "Generic polar harmonic transforms for invariant image description", IEEE conf. on Image Processing, Vol. 6, Issue 6, pp. 617-626 doi:10.1049/iet-ipr.2011.0510.

[12] Bana, S., Kaur, D, 2011, "Fingerprint recognition using image segmentation", International Journal of Advanced Engineering Sciences and Technologies, Vol. 5, Issue No. 1, 012-023.

[13] Lin, H., Si, J, March 2008, "Orthogonal rotation invariant moments for digital image processing", IEEE Trans. on Image Processing, Vol. 17.

[14] Chong, C,W., P. Raveendram., R, Mukundan, 2002, "A comparative analysis of algorithms for fast computation of Zernike Moments", Pattern Recognition Society, Published by Elsivier Science ltd.

[15] Teh, C.H., Chin, R.T., July, 1998, "On image analysis by the method of moments", IEEE Trans. on pattern analysis and machine intelligence, Vol. 4.

[16] Kasza, P., "Pseudo-Zernike Moments for Feature Extraction and Chinese Character Recognition", In Proc. of second International Conference on Computer Automation and Engineering published by IEEE. 
[17] Malathi, S., Maheswari, S.U., and Meena, C, Feb, 2010, "Fingerprint Pore Extraction Based on Marker Controlled Watershed Segmentation", In Proc. of second International Conference on Computer Automation and Engineering published by IEEE.
[18] Rani, U and Ranade, S.K., July 2010, "Improving Accuracy of Pattern Matching Technique with Polar Harmonic Transform for Poroscopy", International Journal of Advanced Research in Computer and Communication Engineering, Vol. 1, Issue 5. 